\title{
A Novel Approach For Sensor Noise Elimination In Real Time Process
}

\author{
K.UMASURESH* ${ }^{*}$ K.SURESH MANIC
}

\begin{abstract}
A review on the current literature on sensor noise elimination algorithms, reports the existence of several adaptive noise reduction strategies. The well known, Wiener filter, which is an optimum filter, belongs to this category and removes the noise, in the mean square error sense. A neural network based independent component analysis (ICA) algorithm is incorporated into the existing system to remove the sensor noise. The proposed intelligent controller, along with this ICA algorithm for sensor noise elimination, can be used to handle plant exceptions, such as poor performance of controller due to faulty sensor readings reduced life of actuators due to frequent false actuating signals to the control valve, to regulate the manipulated variable even when the process is at steady state.
\end{abstract}

Index Terms-Adaptive filter, Independent Component Analysis.

\section{INTRODUCTION}

The algorithms developed in this investigation are tested directly on a nonlinear process, since practical systems are not precisely linear. Even, when represented as linearised models around a nominal operating point, the real system characteristics may not be reflected due to variations in process parameters. A nonlinear (the hopper type tank) multivariable interacting (level and flow) process, with parameters (process gain, process time constant), varying with respect to the process variable level, is considered for real time implementation and study. Even though the process is of the first order and simple, it has the desired severe nonlinearity. A shift in the operating point from the bottom to the top of the tank will alter the process time constant and gain drastically. A deliberate feedback sensor fault is introduced in the sensor used for measuring the process variable level, at random time instants, for a considerably long duration, to test the consistency of the designed estimator. By introducing an artificial sensor noise, the effectiveness of the independent component analysis (ICA) based sensor noise elimination algorithm is easily observed. Since the requisites for testing the algorithms were present in the chosen model the same was taken up for study.

\section{Adaptive Noise CANCELlation Network}

The sensor noise removal algorithm along with the intelligent control algorithms (Karsten Spreitzer et al 2000), belong to the class of noise elimination technique. This network architecture is shown in Figure 1. The delay path serves to generate a reference signal, that can be used to estimate $\mathrm{x}(\mathrm{k})$. It has the advantage, that, it can remove the noise by observing the mixed signal alone. No apriori statistical information about the desired signal is required to be given as input to the network. Also, the source signal $\mathrm{x}(\mathrm{k})$ need not be stationary. This motivates the use of blind signal separation (BSS) algorithms to remove the sensor noise, and extract the true value of the process from the noisy sensor readings. A neural network based independent component analysis (ICA) algorithm is incorporated into the existing system to remove the sensor noise. The proposed intelligent controller, along with this ICA algorithm for sensor noise elimination, can be used to handle plant exceptions such as poor performance of controller due to faulty sensor readings and reduced life of actuators due to frequent false actuating signals to the control valve, to regulate the manipulated variable even when the process is at steady state. This problem arises, since, at steady state $\mathrm{x}(\mathrm{k})$ is stationary but the conventional control algorithms has access only to the noisy sensor readings $\mathrm{s}(\mathrm{k})$.

By using the proposed sensor noise elimination algorithm (ICA network based) at the input of each of the separately located low cost sensors, the noise rejection capability of the sensor is improved at no extra hardware costs.

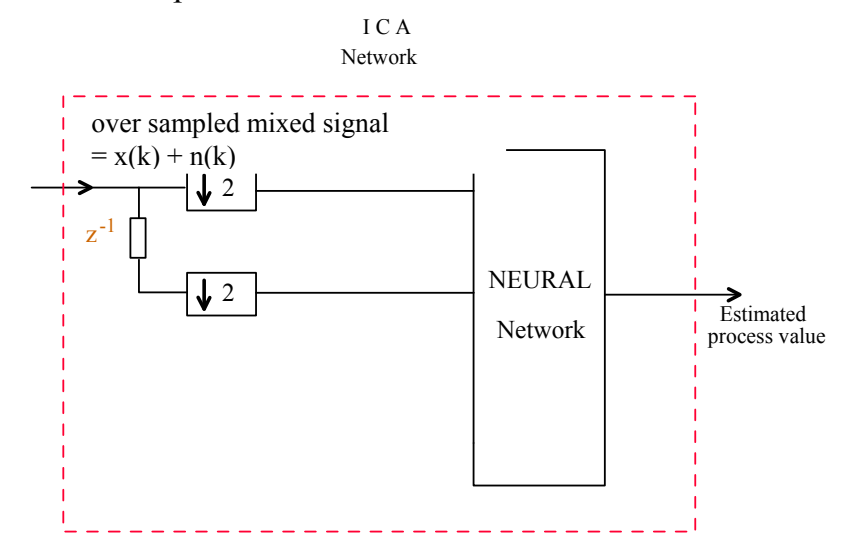

Figure 1 Adaptive noise cancellation network to include non-stationary $\mathrm{x}(\mathrm{k})$ with no secondary noise source.

\section{INDEPENDENT COMPONENT ANALYSIS}

The independent component analysis consists of three steps Whitening, Separation and Estimation. 


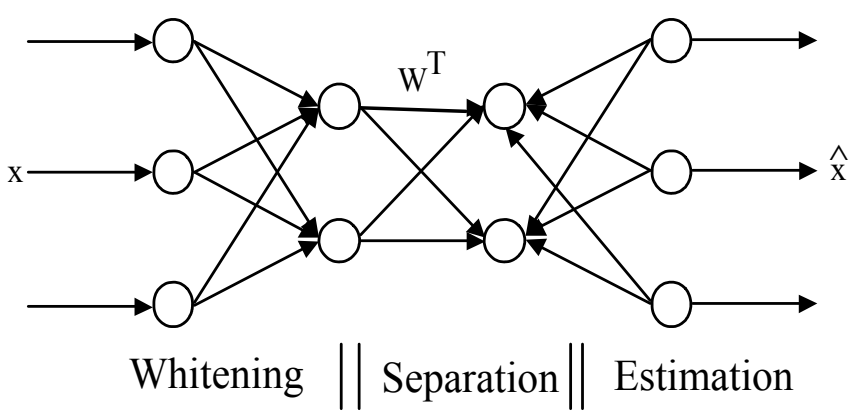

Figure. 2. ICA network used for removing the noise from the sensor readings

If the observed data vectors $\mathrm{x}_{\mathrm{k}}$ have a nonzero mean, then it is desirable to remove the mean. This process is called whitening. The effects of second-order statistics can be removed by using the whitening transformation (Cardoso et al 1997). The whitening matrix is chosen so that the covariance matrix of the whitened vectors $\left\{\mathrm{v}_{\mathrm{k}} \mathrm{v}_{\mathrm{k}}{ }^{\mathrm{T}}\right\}$ equals the unit matrix $\mathrm{I}_{\mathrm{M}}$. After whitening, the components of the whitened vectors $\mathrm{v}_{\mathrm{k}}$ are mutually uncorrelated with zero mean and unit variance.

Uncorrelatedness is a necessary prerequisite for the stronger independence condition, so that after pre-whitening, the noise removal task from the observed mixed data usually becomes easier. There exist infinitely many solutions for whitening the input data, provided that the number of observations of the noisy sensor readings exceeds or atleast equals the number of source signals.

\section{Principal COMPONENT ANAlysis (PCA) BASED WHITENING ALGORITHM}

In this work, the standard PCA (Jutten et al 1997, Karhunen et al 1997, Yanwei Huang et al 2009) based approach is used for whitening. This method has the advantage that it can simultaneously compress information optimally in the mean-square error sense and filter possible noise.

The following steps are used in the PCA based pre-whitening process:

Remove the mean value from the noisy sensor readings $\mathrm{s}[\mathrm{k}]$

Form the covariance matrix of the observed signal $\mathrm{E}\left\{\mathrm{s}_{\mathrm{k}} \mathrm{s}_{\mathrm{k}}^{\mathrm{T}}\right\}$.

Extract the matrices D and E,

where $\mathrm{D}=\operatorname{diag}[\lambda(1), \ldots \ldots, \lambda(\mathrm{M})]$ is a $\mathrm{M} \times \mathrm{M}$ diagonal

matrix,

$\mathrm{E}=[\mathrm{c}(1), \ldots \ldots, \mathrm{c}(\mathrm{M})]$ is a $\mathrm{L} \times \mathrm{M}$ matrix,

$\lambda(i)$ is the $i_{\text {th }}$ largest eigenvalue of the data covariance matrix

$\mathrm{E}\left\{\mathrm{s}_{\mathrm{k}} \mathrm{S}_{\mathrm{k}}{ }^{\mathrm{T}}\right\}$ and $\mathrm{c}(\mathrm{i})$ is the respective $\mathrm{i}_{\text {th }}$ principal eigenvector.

Form whitening matrix $\mathrm{V}$ using $V=D^{-\frac{1}{2}} E^{T}$

Pre-whiten the inputs using the transformation $v[k]=V s[k]$

\section{A. Separation Process}

The second stage in the ICA network is the separation of the source signals. This can be achieved by using suitable higher-order statistics. The separation process can be modeled as a single-layer neural network with an equal number of input and output nodes, where the coefficient $\mathrm{w}_{\mathrm{ij}}$ of the separation matrix $\mathrm{W}$, are simply the weights from the input to output nodes. In the present work, the noise is removed from the measured sensor readings in this stage.

Several types of separation algorithms have been in use, such as the HJ algorithms, PFS algorithm (Cardoso 1996), Bell's and Sejnowski's algorithm (Bell and Sejnowski 1995), bigradient algorithm, nonlinear principal-component-analysis (PCA) subspace learning rule, etc. The nonlinear PCA sub-space learning algorithm is used in the present work, and has the advantage that it can be realized using a simple modification of the one-layer standard symmetric PCA network (Karhunen et al 1995).

\section{B. Neural network based separation algorithm}

The neural network based separation algorithm was implemented by writing a 'c' language program. The pseudo-code of the algorithm is given below.

\section{Step 1:}

While (count $<$ number of training epochs)

\{ while $(\mathrm{i}<$ number of observed samples $)\{$

assign $y[k]=W^{T} v[k]$

$\operatorname{assign} \mu=\frac{1}{\left(\frac{\gamma}{\mu[k-1]+|v[k]|^{2}}\right)}$

assign

$W[k+1]=W[k]+\mu[k]\{v[k]-W[k] g(y[k])\} g\left(y^{T}[k]\right)$

$\mathrm{i}++$

\}

count++

\}

Where $\gamma$ is a constant called the forgetting factor and is chosen as 0.6 ,

$\mu$ is the learning parameter

$\mathrm{W}$ is the separation matrix and

$\mathrm{g}($.$) is a suitable nonlinearity$

Step 2:Estimate the source signals using $y[k]=W^{T} v[k] \sqrt{\text { number.of .observed.samples }}$

\section{Choice of Activation Function ' $g($.$) ' Used in the$ Separation Process}

A proper choice of the activation function $\mathrm{g}($.$) is important$ for effective removal of noise present in the sensor readings. The activation function at the output nodes is used for the training mode only and plays a central role in blind signal separation (BSS). Its nature is defined by objective or contrast or score function. The maximum likelihood approach, (Yang 1999) defines the score function as

$$
g_{i}\left(u_{i}\right)=-\frac{\partial \log P_{s}\left(u_{i}\right)}{\partial u_{i}}=-\frac{p_{s}^{\prime}\left(u_{i}\right)}{p_{s}\left(u_{i}\right)} \quad \mathrm{i}=1, \ldots \mathrm{M}_{\mathrm{s}}
$$


where $\mathrm{p}_{\mathrm{s}}\left(\mathrm{u}_{\mathrm{i}}\right)$ and $\mathrm{p}_{\mathrm{s}}^{\prime}\left(\mathrm{u}_{\mathrm{i}}\right)$ are the probability density function (pdf) and its derivative, respectively, of the source signals. In this work, a sigmoidal activation function of the form $\tanh ($. is chosen.

\section{Multiple Sensor Coordination Model (With SENSOR NOISE ELIMINATION SCHEME AND FEEDBACK SENSOR VALIDATION NETWORK)}

The nonlinear plant with the noise added to both the inflow and outflow measuring sensors is shown in Figure 3. The multiple sensor coordination model proposed for use in an interacting multivariable nonlinear system, to accommodate single sensor fault and noise in the measured states, that may occur independently or simultaneously (Demetrious et al 1998), is shown in Figure 4. a,b. The two independent ICA networks are used to remove the noise present in the inflow and outflow sensors, while the neural network or fuzzy trained estimator takes care of the feedback sensor failure.

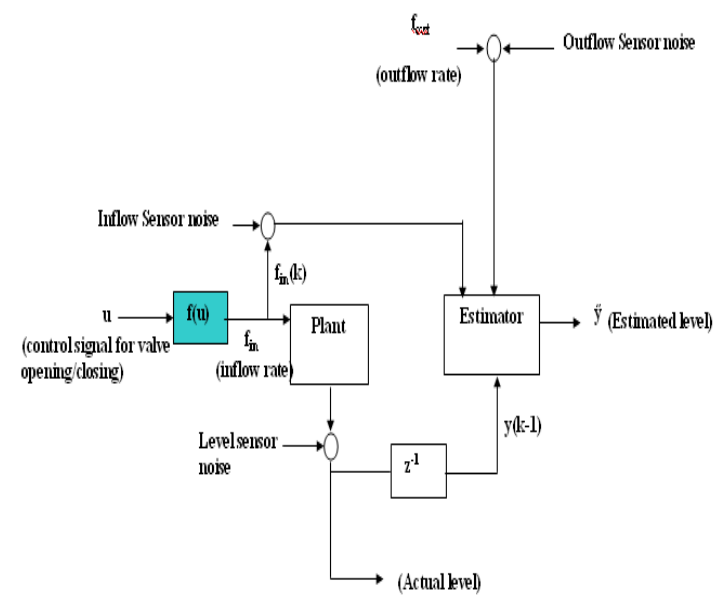

Figure 3. Plant model with sensor noise added to inflow and outflow sensors

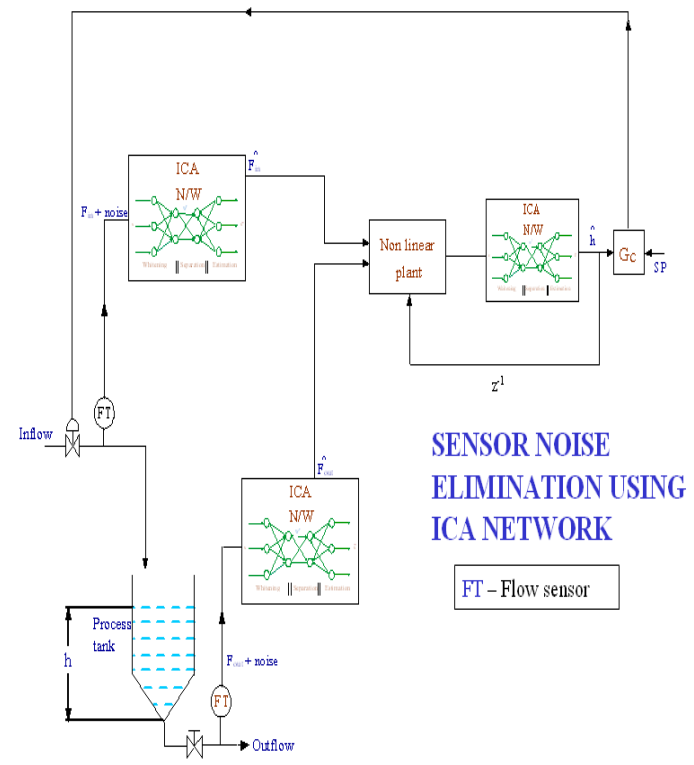

Figure 4.a. multiple sensor coordination model with the ICA network for sensor noise elimination in a multivariable interacting system

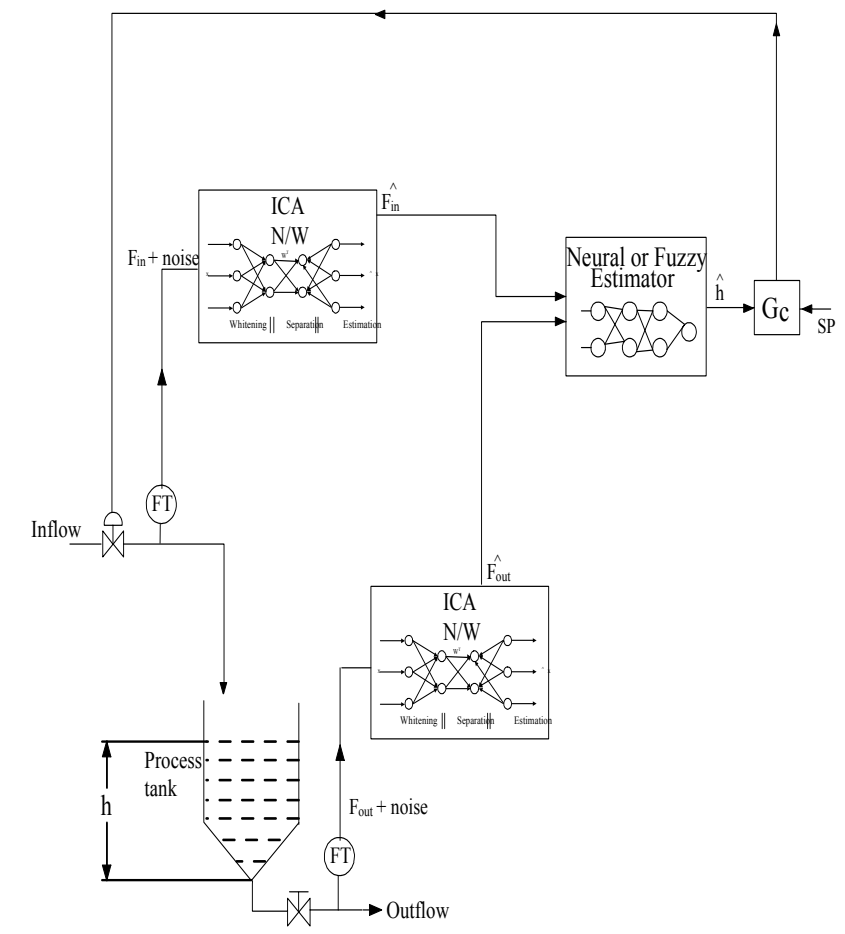

Figure 4.b. Multiple sensor coordination model with the ICA network for sensor noise elimination and neural network or fuzzy trained estimator for feedback sensor validation in a multivariable interacting systems

\section{EXPERIMENTAL RESULTS}

The focus of this work is to demonstrate the capability of the designed noise elimination algorithm, to remove the sensor noise and extract the true value of the process, independent of the ensemble statistics of a signal. The distribution of the noise model is important since it is a representative of the physical source from where the noise originated (Adler et al 1998). In the present work, the sensor noise was created during each of the trial, by filtering noise samples initially drawn from a Gaussian distribution. Though the proposed algorithm is designed to remove noise (Srinivasan et al 2006) without regard to a particular distribution, the noise was assumed to have evolved from a Gaussian distribution, since it is sensible in terms of the central limit theorem .

Samples of the non-stationary signals inflow, outflow and level of sufficiently long duration was captured for study and many trials were performed. The samples were quantised with an 8-bit resolution (Zhang et al 2002). The noisy sensor readings were over sampled, and presented to the noise elimination network of Figure 5.3. It was assumed throughout the period of study that the autocorrelation sequence of the signal can decay to zero at a slower rate than that of the noise. This assumption is reasonable and is typical of the one that would occur in a real time process control plant. The whitening and separation algorithms (section 5.1) were used, and an estimate of the true value was obtained. The mean square error (MSE) criterion was used as a measure of performance index in each case. The normalized MSE was calculated as 
$M S E=\frac{1}{N} \sum_{k=1}^{N}[$ true value of the measured state estimated value $]^{2}$

where $\mathrm{N}$ is the number of samples and is equal to 400 .

\section{A. Eliminating level Sensor Noise (For Servo Tracking)}

The true variations of the process variable level, the observed noisy sensor values and the ICA estimated level variations, for this case are shown in Figures 5.1 to 5.4 respectively.

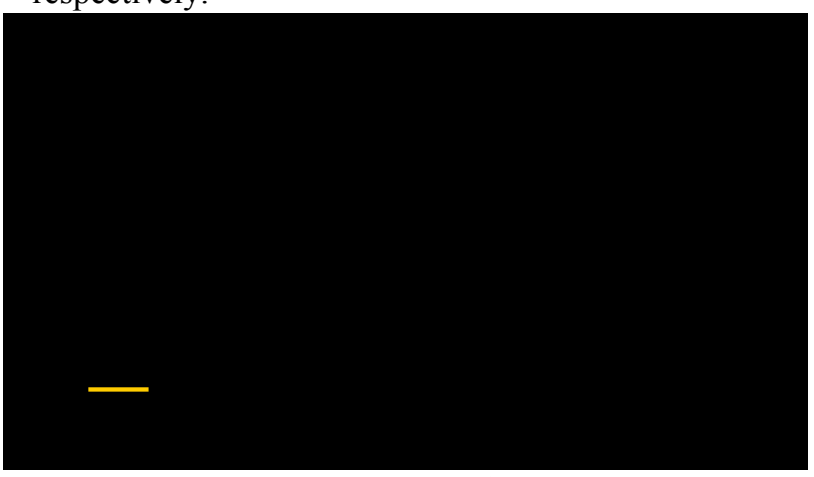

Time samples

Figure 5.1. magnified view of the true variations of process level in the experimental servo tracking response with FLC (i.e. level variations with sensor noise $=0$ )

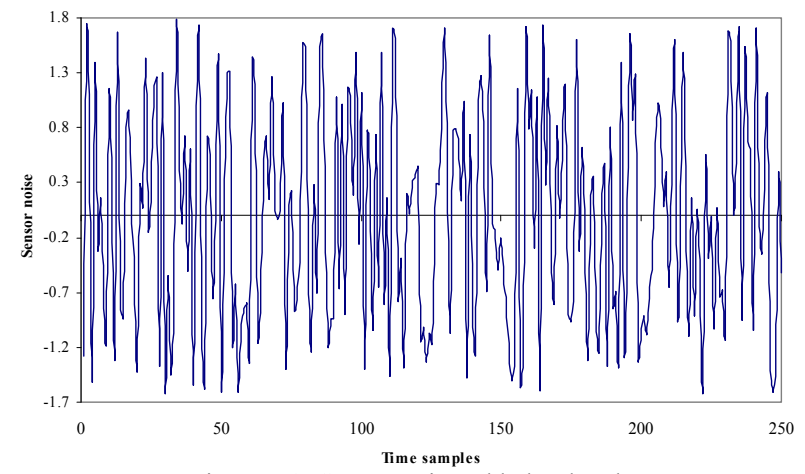

Figure 5.2. Sensor noise added to level

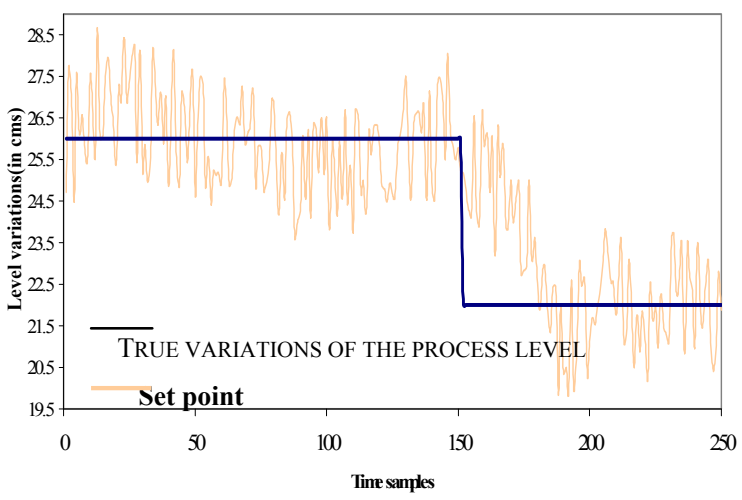

Figure 5.3.Magnified view of the true variations of process level in the experimental servo tracking response with FLC (i.e. level variations with sensor noise $=0$ )

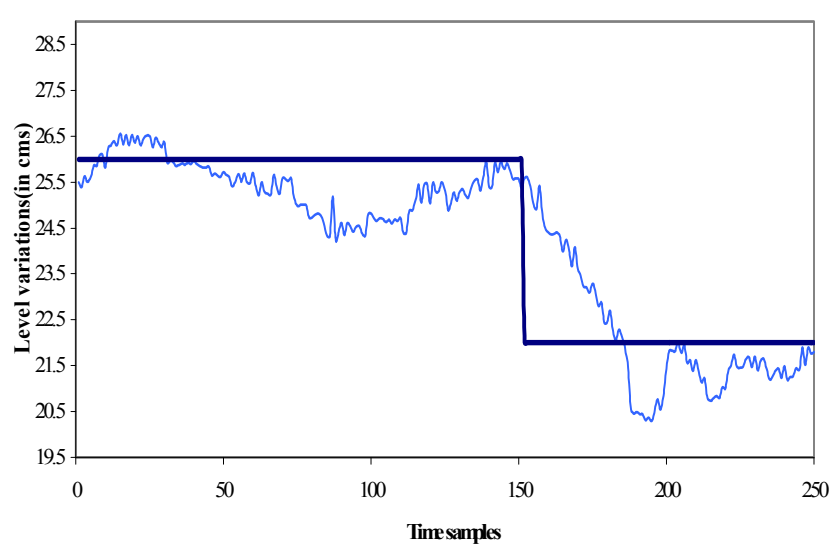

Figure 5.4.Magnified views of the estimated variations of process level in the experimental servo tracking response with fuzzy controller

\section{B. Eliminating level Sensor Noise (For load variation)}

The true variations of the process variable level, the observed noisy sensor values and the estimated level variations, for this case are shown in Figures 5.5 to 5.8 respectively.

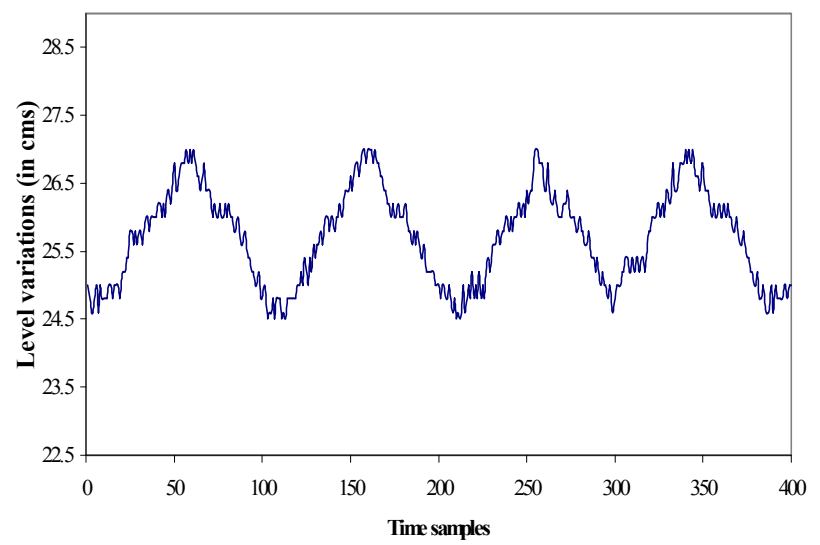

Figure 5.5.Magnified view of the true variations of process level in the experimental regulatory response (response in presence of perturbations in load variable outflow and with sensor noise $=0$ )

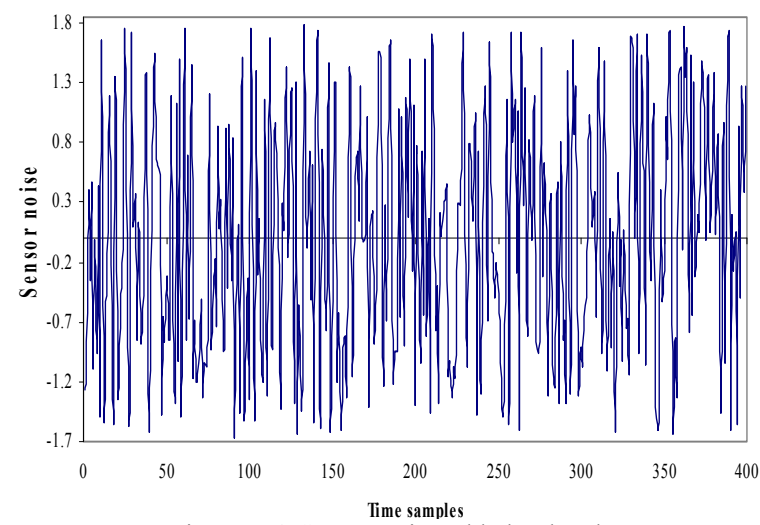

Figure 5.6. Sensor noise added to level 


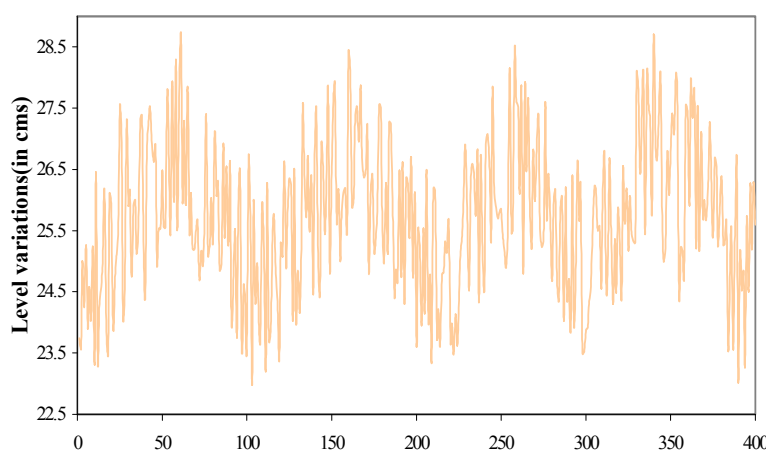

Time samples

Figure 5.7 .Magnified views of the variations of process level in the experimental regulatory response in the presence of sensor noise

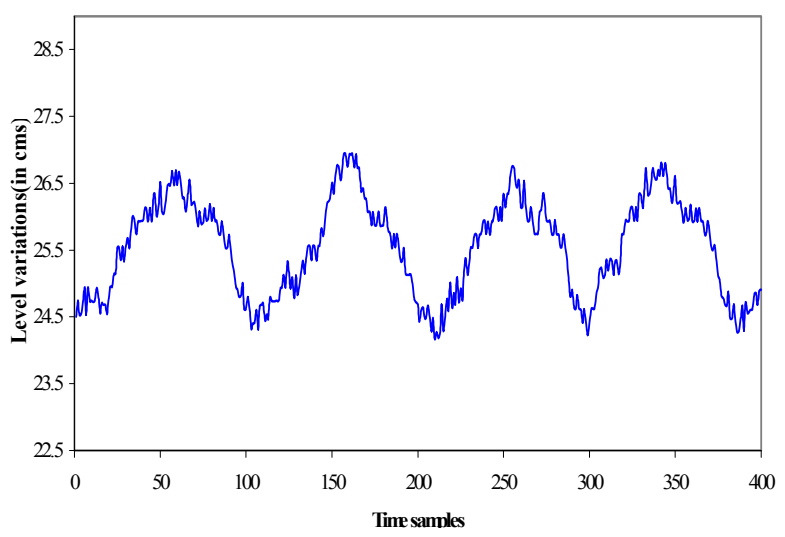

Figure 5.8.Magnified views of the estimated variations of the process level in the experimental regulatory response

\section{Eliminating Inflow Sensor Noise}

The true value of the inflow rate, added sensor noise, the observed noisy sensor readings and the estimated inflow variations for this case are shown in Figure 6.

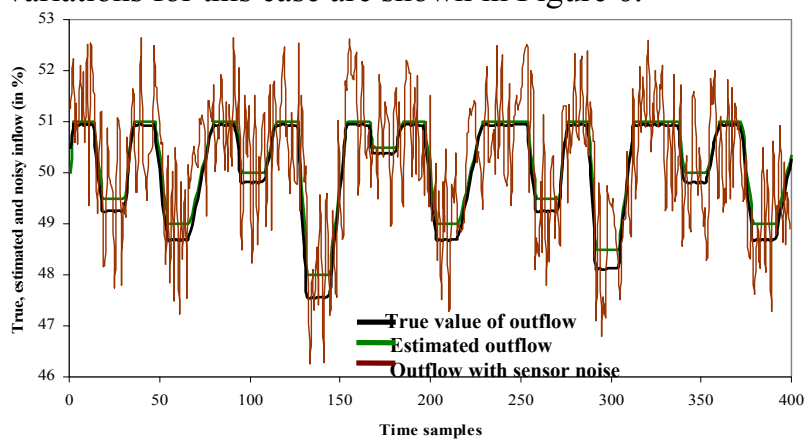

Figure 6. Combined plot of true fluctuations in inflow, noisy sensor values and the estimated inflow (in \%) about its nominal value of $50 \%$

\section{Eliminating Outflow Sensor Noise}

The true value of the load variable outflow, added sensor noise, the observed noisy sensor readings is shown in Figure 7 .

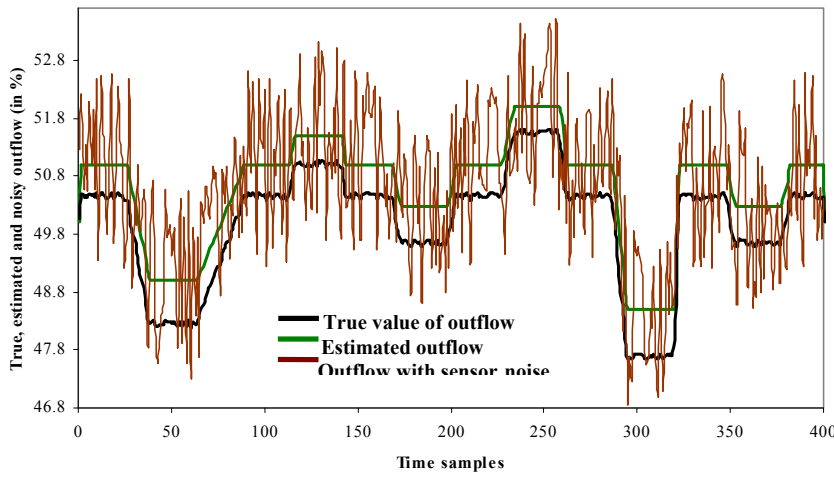

Figure 7. Combined plot of true fluctuations in outflow, noisy sensor values and the estimated outflow (in \%) about its nominal value of $50 \%$

\section{ON-LINE ACQUIRED PLOTS}

The on-line acquired plots for set point and load tracking during process outflow, ICA estimated value and process value with noise is shown in figure 8 and 9 .

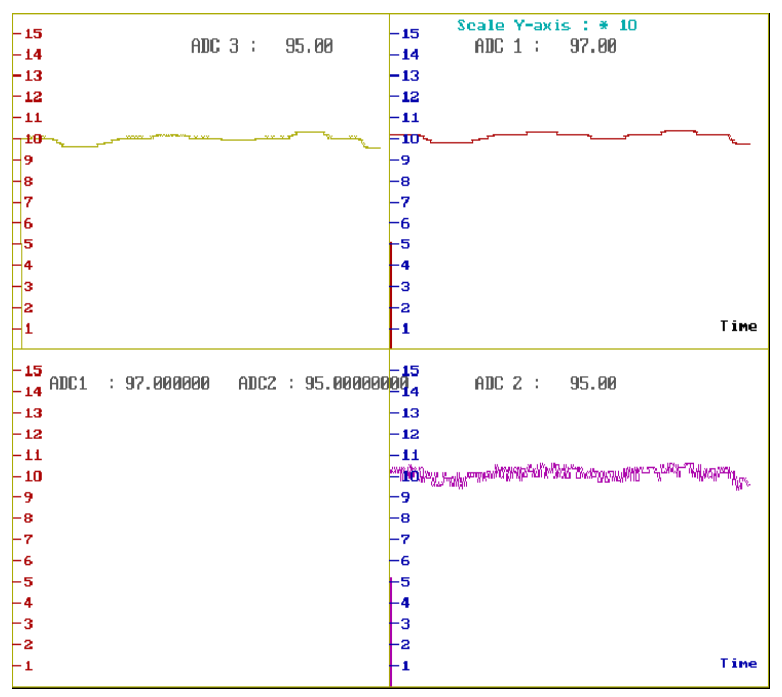

Figure 8. On-line acquired plots showing the true process value of outflow (1st quadrant), ICA estimated value (2nd quadrant) and process value with noise (4th quadrant) (Plot obtained during load tracking)

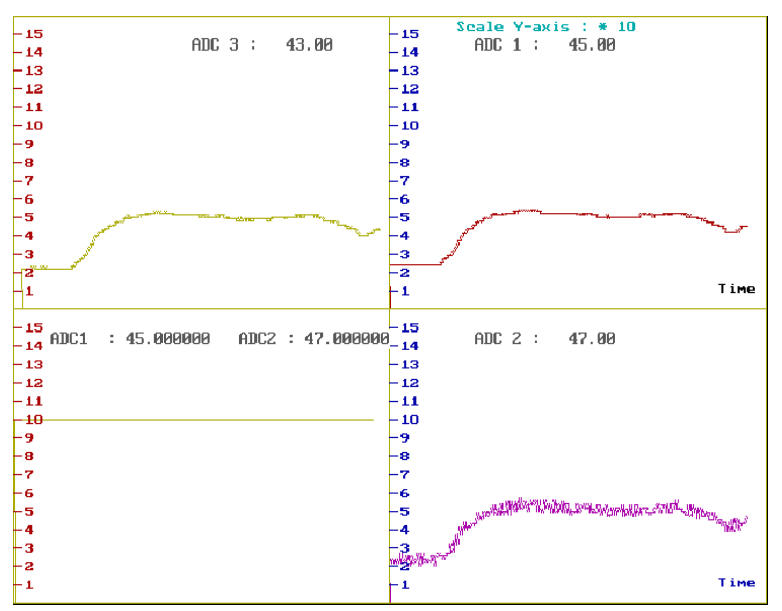

Figure 9. On-line acquired plots showing the true process value of level (1st quadrant), ICA estimated value (2nd quadrant) and the process value with noise (4th quadrant). (Plot obtained during set point tracking) 


\section{Discussion on the Performance Of The Sensor NOISE ELIMINATION NETWORK}

The proposed sensor noise elimination scheme was implemented and tested for satisfactory performance on the real time plant. The true sensor readings were corrupted with Gaussian noise (Mhaskar et al 2007) and presented to the sensor noise elimination network. The estimated value is compared with the true value, by computing the mean square error deviation (MSE). From the obtained MSE value, it can be concluded that the estimated values matches well with the true values of the measured states. In particular, the ability of the network to process the noisy level sensor readings and give a good estimate of the true value during the servo tracking response is significant.

The proposed sensor noise elimination algorithm can be incorporated with ease, into any of the existing process control loops, at no extra hardware costs (Mufed Mohmoud et al 2001). This permits the use of a commercially available low cost sensor, with the sensor noise elimination taken care by the proposed algorithm. The algorithm can be implemented easily using the unsupervised neural learning architecture. The algorithms are designed to remove the sensor noise in a manner ideally suited for a real time environment, where the sensor noise elimination process need to be performed independent of the ensemble statistics of the signal without regard to a particular class of signal model. The MSE values obtained for the different cases are summarized in chapter 6 .

However, it should be mentioned here, that in the area of blind signal separation, the theoretical properties, range of applicability and mutual comparisons still remains largely unexplored.
[11] Mufeed Mahmoud, Jin Jiang and Youmin Zhang (2001), 'Stochastic stability analysis of fault-tolerant control system in the presence of noise', IEEE Transaction on Automatic Control, Vol. 43, pp.1617-1621.

[12] Srinivasan K. and Prakash J. (2006), 'Non-linear state estimation for continous stirred tank reactor using neural network state filter', IEEE Transactions.

[13] Yang H.H. (1999), 'Serial updating rule for blind separation derived from the method of scoring', IEEE Trans. Signal Processing, Vol. 47, pp.8

[14] Yanwei Huang and Xianbo Qiu (2009),'Fault diagnosis for dynamic Nonlinear system based on Kernal principal component analysis', IEEE Transaction on Computer society, pp 680-683.

[15] .Zhang X, Polycarpou M.M. and Parisini T. (2002),' A robust detection and isolation scheme for abrupt and incipient faults in nonlinear systems', IEEE Transactions on Automatic Control, Vol. 47, No. 4, pp. 576-593.

K. Uma Suresh is currently a P.G Student (Embedded System Technology) in the Department of Electronics \& Communication Engineering, at Sriram Engineering College, Chennai. Her areas of interest include embedded system design, controller design.

K. Suresh Manic is presently a Prof of Electrical Engineering at Sriram Engineering college, Afilliated to Anna University, Chennai. His areas of interest include fault tolerant system design, Fuzzy controller Evolvable hardware, process control.

\section{REFERENCES}

[1] Adler R.J., Feldman R.E. and Taqqu M.S. (1998), 'A Practical Guide to Heavy Tails; Statistical Techniques and Applications', Boston, MA: Birkhauser.

[2] Bell A. and Sejnowski T. (1995), 'An information-maximisation approach to blind separation and blind deconvolution', Neural Computation, Vol. 7, pp. 1129-1159.

[3] Cardoso J.F. (1996), 'The Invariant approach to Source Separation', Proc. 1995 Int. Symp. on Non linear theory and Applications (Nolta-95), Las Vegas, USA.

[4] Cardoso J.F. and Laheld B. (1997), 'Equivariant Adaptive Source Separation', IEEE Trans. on Signal Processing.

[5] Demetrious M.A. and Polycarpou M.M. (1998),'Incipient fault diagnosis of dynamical systems using online approximators', IEEE Transaction on Automatic Control, Vol. 43, pp.1612-1617.

[6] Jutten C. and Cardoso J.F. (1997), 'Separation of Sources: really blind', in Proc. 1995 Int. symp. on Nonlinear Theory and Applications (Nolta-95), Las Vegas, USA

[7] Karhunen J., Wang L. and Vigario R. (1995), 'Nonlinear PCA type approaches for souece separation and independent component analysis', in Proc. 1995 IEEE Int. Conf. on Neural Networks, Perth, Australia, pp. 995-1000.

[8] Karhunen J., Oja E., Wang L., Vigario R. and Joutsensalo J. (1997), ‘A class of Networks for Independent Component Analysis', IEEE Trans. On Neural Networks, Vol.8, pp. 486-504.

[9] Karsten Spreitzer and Peter Balle (2000),'A multi-model approach for detection and isolation of sensors and process faults for a heat exchanger', Proceedings of the American Control Conference, pp. 2730-2735.

[10] Mhaskar P., Gani A., McFall C., Christofides P.D. and Davis J.F. (2007), 'Fault tolerant control of nonlinear process systems subject to sensor faults', AIChE J., Vol. 53, pp 654-668. 